Wagner, K., \& Barner, D. (in press). The acquisition of color words: How children perceive, abstract, categorize, and label color. Linguistics: Oxford Research Encyclopedias.

\title{
The Acquisition of Color Words
}

\author{
Katie Wagner and David Barner \\ University of California, San Diego \\ Psychology Department
}

Corresponding author:

Katie Wagner

kgwagner@ucsd.edu

University of California, San Diego

Psychology Department

9500 Gilman Dr.

La Jolla, CA 92093 


\section{Summary}

Our experience of color results from a complex interplay of our perceptual and linguistic systems. At the lowest level of perception, our visual system transforms the visible light portion of the electromagnetic spectrum into a rich 3D experience of color. Despite our ability to discriminate millions of different color shades, most languages categorize color into discrete color categories. Perception provides constraints on the likely locations of color word boundaries but does not fully define color word meanings. Once acquired, although language likely does not influence the lowest levels of color perception, language does influence our memory and processing speed of color.

One approach to examining the relationship between perception and language in forming our experience of color is to study children as they acquire color language. Children produce color words in speech for many months to years before acquiring adult-like meanings for color words. Research in this area has focused on whether children's difficulties stem from 1) an inability to identify color properties as a likely candidate for words meanings or alternatively 2) inductive learning of language specific color word boundaries. Supporting the first account, there is evidence that children more readily attend to object traits like shape rather than color as likely candidates for word meanings; however, children seem to have successfully identified color a candidate for word meaning before they begin to produce color words in speech. There is also evidence that pre-linguistic infants, like adults, perceive color categorically. While these perceptual categories likely constrain the meanings that children consider, they cannot fully define color word meanings because languages vary in both the number of location of color word boundaries. Recent evidence suggests that the delay in color word acquisition primarily stems from an inductive process of refining these boundaries. 


\section{Keywords}

Color language; Color perception; Word learning; Category learning; Language acquisition; Linguistic variability 
Our experience of color is a complex response to the variability in wavelengths of the visible electromagnetic spectrum. Together, our eyes and brain transform this one dimensional signal into a three dimensional color space that in some ways is perceived continuously and yet in other ways, is perceived categorically. We can perceive and discriminate numerous shades between the color of blueberries and that of the sky but nevertheless label all of these colors blue. Yet, if we shift the color of blueberries only slightly, the resulting shade resembles the color of eggplants and we label it purple.

This phenomenon points to a problem that humans must solve: Why are some aspects of color perception categorical, but not others? And how do words like blue and purple map onto perception? Are words purely a system of labels that map onto existing perceptual distinctions e.g., windows to perception (Barner, Li, \& Snedeker, 2010)? Or do words transform perception when we learn them, leading to differences between speakers of different languages, and between children at different stages in language acquisition?

One way to answer these questions is by exploring how color perception emerges in human infants, and whether perception changes as a function of culturally variable linguistic experience. Interestingly, children appear to struggle to learn color words, and use them in a seemingly random fashion for many months before converging upon adult-like meanings. The problem of how children learn color word meanings is an active area of research, closely tied to the controversial question of how language and color perception are related. Here we provide a brief review of color perception and a discussion of the current state of research into how humans acquire a vocabulary to describe this perceptual experience.

\section{Overview of lower level color perception}

\section{Humans have three types of daytime photoreceptors}


Light is first converted into a neurological signal at our retina via cells called photoreceptors. Photoreceptors called rods dominate nighttime vision and photoreceptors called cones dominate daytime vision and are critical to color perception.

The number and type of photoreceptors that a species has influences how they perceive color. Most humans, Old-world primates and many marsupials are trichromats, who perceive color along three dimensions as a result of possessing three distinct types of color cones (for review see Jacobs, 2009). However, most other land mammals are dichromats (Jacobs, 2009), and have only two types of color cones (as do $8 \%$ of human males and 0.5 percent of human females with the most common types of color deficiency, for review see Simunovic, 2010). Many sea mammals have even less rich color vision, as they typically have only one type of cone and consequently perceive color along a single monochromatic dimension (Jacobs, 2009). On the other end of the spectrum, the mantis shrimp has 16 types of photoreceptor (Marshall \& Oberwinkler, 1999; Cronin \& Marshall, 1989; Cronin, Bok, Marshall \& Caldwell, 2014). This variability in how light is encoded has profound perceptual consequences. For example, to a human observer a light brown deer is not well camouflaged in a green meadow. To dichromatic predators that hunt deer, however, the deer blends right in.

Human cones are sensitive to light with wavelengths between $400 \mathrm{~nm}$ and $700 \mathrm{~nm}$. This makes us less sensitive to longer wavelengths than some species - e.g., reptiles (Goris, 2011), and less sensitive to short wavelengths than other species - e.g., the mantis shrimp and some insects (Briscoe \& Chittka, 2001; Marshall \& Oberwinkler, 1999). The 


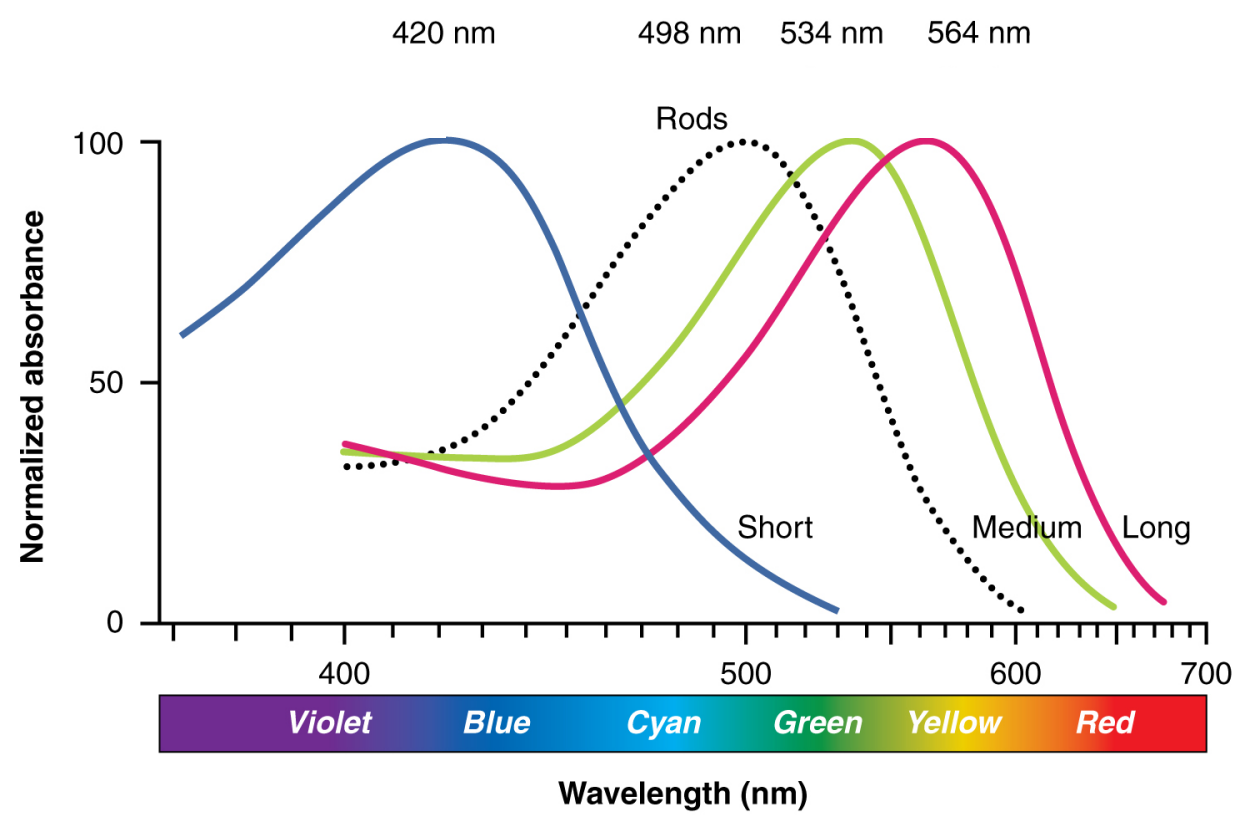

Figure 1. Displays the peak absorption and range of absorption for each of the three photorecptors responsible for daytime vision (cones) and the one type of photoreceptor responsible for night time vision (rods).

Edited by authors. Original by OpenStax College [CC BY 3.0 (http://creativecommons.org/licenses/by/3.0)], via Wikimedia Commons portion of the electromagnetic spectrum that humans are sensitive to is called visible light. Importantly, we are not equally sensitive to every part of the visible light spectrum. Each of the three different types of cones contains a different type of photopigment that is sensitive to a different part of the visible light spectrum, see Figure 1. Short wavelength cones have a peak absorption rate around 430nm (e.g., for review, see Teller \& Bornstein, 1987) and are relatively sparse, on average accounting for only about $5-8 \%$ of cones in the human retina (Curcio et al., 1991; Hofer, Carroll, Neitz, Neitz \& Williams, 2005; Roorda \& Williams, 1999). The remainder of the cones are medium wavelength cones with peak absorption occurring around $530 \mathrm{~nm}$ and long wavelength cones with peak absorption occurring around $560 \mathrm{~nm}$ (for review, see Teller \& 
Bornstein, 1987). In color normal individuals, the proportion of both medium or long wavelength cones is much greater than the proportion of short wavelength cones, though there is a high degree of individual variability in the relative proportion of these two cone types (Hofer et al., 2005; Roorda \& Williams, 1999). Due to the higher density of long and medium wavelength cones and their overlapping sensitivity to the middle range of the visible light spectrum (see Figure 1), humans are most sensitive to and can detect finer grain differences to light with wavelengths in mid-range of the visible spectrum (e.g., yellows and greens) and have lower sensitivity to light comprised of longer wavelengths (e.g., reds) or shorter wavelengths (e.g., purples). As a result, even at the lowest levels of color perception, our ability to detect differences between two colors is not linearly related to the physical differences between colors as measured in wavelength. Instead, our sensitivity to differences between colors is highly dependent on where these colors are situated in color space.

\section{Opponent color space}

Low level color processing is best characterized by opponent color space. Opponent color space is the product of three distinct visual pathways. These are the magnocellular (light-dark) pathway, the parvocellular (red-green) pathway, and the koniocellular (blue-yellow) pathway. Each pathway begins with the retinal ganglion cells that receive input from the three types of color cones discussed above. The magnocellular pathway combines input from the long and medium cones in an additive way, so that a larger response from either of these two cones indicates a brighter color. The parvocellular pathway combines data from the long and medium cones in a subtractive way. A redish perception occurs when activation of the L-cone is greatest relative to the M-Cone, and a greenish perception occurs when activation of the M-cone is greatest relative to the L-cone, see Figure 1. Finally, the parvocellular pathway receives input 
from all three cone types. The combination of L-cone and M-cone activation is combined with Scone activation in a subtractive way. When S-cone activation is greatest relative to $\mathrm{L}+\mathrm{M}$ cone activation, we experience a bluish perception, and when S-cone activation is lowest relative to $\mathrm{L}+\mathrm{M}$ cone activation, we experience a yellowish perception. For review, see Wässle, 2004; Teller \& Bornstein, 1987).

\section{Color Constancy}

While opponent color space represents subcortical color processing well, additional factors are required to explain conscious color perception. For example, our day-to-day experience of color exhibits "color constancy". Color constancy refers to the phenomenon whereby objects are perceived to have the same color under different lighting conditions that result in radically different wavelengths. The spectrum of wavelengths that characterize daylight, for example, varies depending on the time of day and the season. However, most humans remain unaware of this fluctuation because of our visual system's remarkable ability to adjust for ambient lighting. As a result of this, a particular apple will typically appear to be the same shade of red whether it is viewed under the yellow fluorescent lights of a grocery store, or under 
daylight at different times of day. See Figure 2 for a demonstration of this phenomenon.

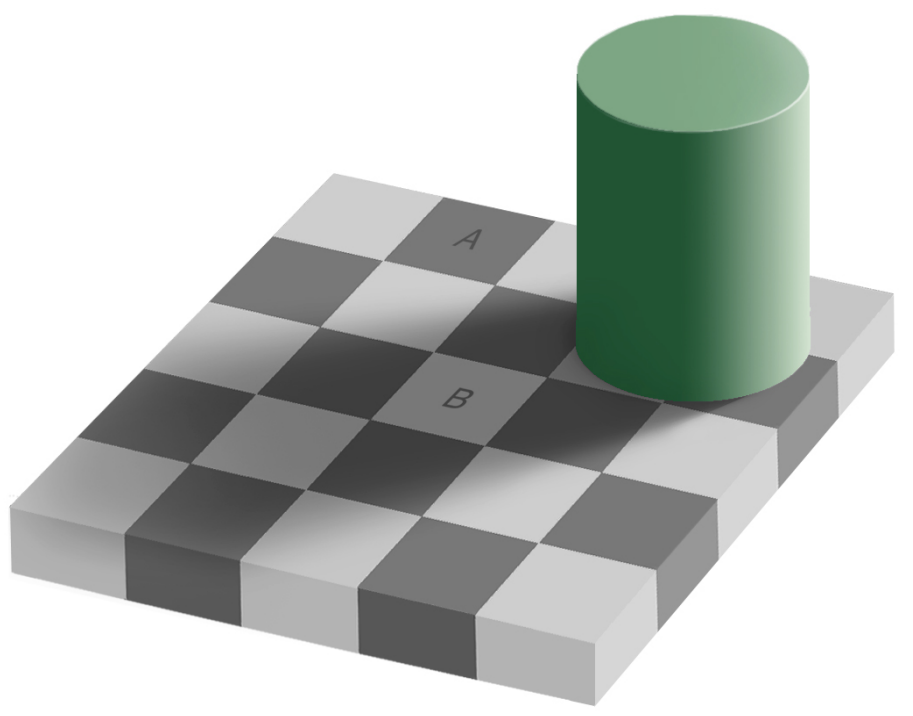

Figure 2: Squares $A$ and $B$ reflect the same amount of light. However, $B$ appears lighter because our visual system takes into account that it is sitting in the shadow of the cylinder. Therefore, $B$ 's true color (the proportion of the light that it reflects) is likely to be lighter than $A$ given that they are reflecting the same absolute quantity of light. This can be proved by covering the space between the shadowed and non-shadowed areas.

Original by Edward H. Adelson, this file by Gustavb [Copyrighted free use], via

\section{Wikimedia Commons}

\section{The development of color perception.}

Studies dating back at least 100 years have observed that color words pose a difficult problem to children learning language (e.g., Bateman, 1915; Nagel, 1906). Although children now learn color words earlier, reports in the early $20^{\text {th }}$ century found that children learned them as late as age 8 (Bateman, 1915). Some initial explanations of this delay suggested that children were slow to learn color words because color vision itself was slow to develop. In fact, due to the immaturity of their color cones, retinas, and color pathways, newborns are very nearsighted, 
have poor visual acuity, and a very limited ability to discriminate between different colors (for review, see Dobson \& Teller, 1978; Boothe, Dobson \& Teller, 1985; Teller \& Bornstein, 1987). However, visual acuity and low level color perception develop rapidly in the first six months, and although they continue to develop slowly throughout childhood, they approach maturity by six to twelve months of age (for review, see Dobson \& Teller, 1978; Boothe, Dobson \& Teller, 1985; Teller \& Bornstein, 1987; Knoblauch, Vital-Durand \& Barbur, 2001). Thus, these particular perceptual factors cannot likely explain children's delay in acquiring color words at two and three years of age.

Another perceptual factor that might limit color word learning is constancy. It would be very difficult to learn the meaning for red, for example, if the appearance of red objects fluctuated depending on the time of day and whether the object was indoors or outdoors. However, although very young infants may lack color constancy, color constancy has been observed in infants as young as 5 to 7 months of age (Yang, Kanazawa, Yamaguchi \& Kuriki, 2013; Dannemiller, 1989). Thus, it seems likely that by the time children begin learning color words two years later, color constancy, like low level color perception, is close to that of adults and unlikely to interfere with their acquisition of color words.

\section{Categorical perception of color}

\section{Categorical perception of color in adults}

There is substantial evidence that subcortical color processing is adult-like within the first year of life; however, it is less clear whether or not categorical perception of color is adult-like before children begin learning color words. In fact, there remains debate as to whether categorical perception occurs at the perceptual level (e.g., Heider, 1972) or if it is a phenomenon 
of higher-level cognitive systems, perhaps a result of color language itself (e.g., Roberson, Davidoff, Davies \& Shapiro, 2004).

Categorical perception is not restricted to the domain of color, but refers to a more general phenomenon wherein our perceptual experience imposes categorical boundaries on continuous perceptual information. Perhaps the most well-known example of categorical perception is in the domain of speech (e.g., Eimas, Siqueland, Jusczyk \& Vigorito, 1971; Werker, Gilbert, Humphrey \& Tees, 1981). Adult speakers of English distinguish the phonemes /ba/ and /pa/ according to their "voice onset time" or VOT - i.e., the time it takes for the vocal chords to begin vibrating after the release of a "stop" consonant, like /b/ or /p/, which fully impedes the passage of air. Although it is possible to create a continuum of voice onset times (VOT) between /ba/ and /pa/, all stimuli with a VOT below a certain threshold are perceived as /ba/ and are indistinguishable from each other, and all the stimuli above this threshold are perceived as $/ \mathrm{pa} /$ and are also indistinguishable. Consequently, a pair of stimuli that cross this VOT boundary are perceived as very distinct but a pair of stimuli that differ by the same delta VOT but do not cross the boundary will be indistinguishable.

Categorical perception of color is somewhat more complex. In the case of phonetic categorical perception, above, we discussed category boundaries as they relate to a continuum of physical stimuli. In the case of color, one might assume that things are different, given that humans can discriminate many shades of color within a given color category and given that the the human visual system is not equally sensitive to all wavelengths of light. As already noted, we are most sensitive to mid-spectrum wavelengths and less sensitive to short and long spectrum wavelengths. However, while this non-linear sensitivity compresses the electromagnetic spectrum non-uniformly, this is not the source of categorical perception of color as it is typically 
discussed in the color perception literature. Instead, researchers who study categorical perception of color generally explore color category boundaries as they relate to a continuum of low-level color perception, which is already distorted by our visual system, rather than as they relate to a continuum of physical stimuli. As an example, participants respond faster to a difference in color that crosses category boundaries (a blue and a green) than to a difference in color that are within a color boundary (two shades of blue), even if the difference between the colors in each pair is equated for discriminability in perceptual color space (e.g., Roberson, Hanley \& Pak, 2009).

While the categorical nature of high-level color processing is not disputed, the origin and nature of these color categories remains a controversial research topic. The most frequently studied question regarding color categories is whether they exist independent of language or whether they are imposed on perception by language. Supporting the idea that language imposes categories on perception are data demonstrating categorical responses to color that cross the same boundaries marked by linguistic color categories. Also, the location of categorical boundaries appears to vary according to the language of participants (e.g., Roberson, Hanley \& Pak, 2009; Winawer, Witthoft, Frank, Wu, \& Borodisky, 2007). Finally, in many cases, categorical effects disappear when participants are asked to engage in a concurrent linguistic task (Winawer et al., 2007; Roberson \& Davidoff, 2000, though see Pilling, Wiggett, Özgen, \& Davies, 2003, for a counterpoint).

Although there is substantial evidence for a moderate version of the hypothesis that language influences color categorization, this should not be interpreted as evidence that speakers of languages with more color words are able to see finer differences between colors. If this were the case, we would expect that speakers with separate words for blue and purple, for example, would have smaller Just Noticeable Differences (JNDs) across this boundary than speakers 
without separate words for blue and purple. JNDs measure the smallest amount of change in a color necessary before a participant can reliability tell the two colors apart. Roberson, Hanley, and Pak (2009) compared JNDs in the blue/region of color space between speakers of Korean (which divides this region into three categories) and English (which divides this region into two categories). They found no cross-linguistic differences in JNDs, despite finding that language facilitated accuracy on a color discrimination task which presented the stimuli for only $350 \mathrm{~ms}$. In other words, they found that language does not help participants make finer grained discriminations between colors, despite helping them make these discriminations more quickly (as in Roberson, Hanley, and Pak, 2009).

An alternative to the claim that language affects color categorization is the hypothesis that linguistic color categories arise from perception. This view initially gained support when Berlin and Kay (1969) began mapping out the focal regions and boundaries of linguistic color categories cross-linguistically. By 2009, they had such data from monolingual speakers of 110 unwritten languages (see World Color Survey: Kay, Berlin, Maffi, Merrifield, \& Cook, 2009). Although languages vary with respect to the number of color words they have, Berlin and Kay found evidence that challenged the previous idea that languages divide color space into arbitrary categories. Instead, they found that the focal regions of linguistic color categories clustered in particular areas, and that some boundaries were much more common than others. On the basis of this, they argued that languages follow predictable stages of color word evolution, such that the specific colors encoded by a language are predictable from the number of color words it has.

Further support for this universalist view comes from data comparing speakers of Dani, a language with two basic color words and English, eleven basic color words (Heider, 1972; Heider \& Olivier, 1972; though see Roberson, Davies, \& Davidoff (2000) for a counterpoint). 
Heider found that despite only have two color words, speakers of Dani were better able to remember colors that were focal or prototypical to English color word categories than those that were non-focal. As further evidence, Dani speakers were more accurate at learning new labels for focal colors. In sum, there is evidence that despite cross-linguistic differences in color word categories, these categories and their focal regions are not determined arbitrarily by languages. Instead, there are constraints that inform which category structures are most likely to be highlighted by human language.

\section{Categorical perception of color in infants}

While adult data provide one window into understanding categorical color perception, another approach is to test human infants, who lack extensive experience with language. Specifically, by testing infants, it is possible to ask whether perceptual color categories emerge before labels are learned, and whether labels have any effect on perception once they are acquired.

Providing plausibility to the universalist hypothesis, the current consensus is that infants do possess perceptual color categories well before they begin learning color language. Tests of categorical perception of color in infants have used one of two methodologies: habituation and target-detection. In habituation designs (Franklin \& Davies, 2004; Bornstein, Kessen \& Weiskopf, 1976), infants are repeatedly shown a color until their looking time towards that color decreases. At this point, a new color is introduced that is either in the same color category or a different color category. Infants increase their looking time (or dis-habituate) only to a change of stimulus that involves a change in color category, indicating categorical perception of color. In target-detection tasks (Franklin, Pilling \& Davies, 2005; Franklin, Drivonikou, Clifford, Kay, Regier \& Davies, 2008), experimenters measure how long it takes participants to visually fixate a 
color target that is either in the same color category or different color category as the background color. Consistent with categorical perception of color, infants (Franklin, Pilling \& Davies, 2005) and toddlers (Franklin et al., 2008) who have yet to learn their color terms are faster to fixate the between category than within category target. Importantly, neither the habituation nor target fixation results can be explained by a larger change in lower level color perception on the between category trials (see Franklin \& Davies, 2004; Franklin, Pilling \& Davies, 2005 for discussion).

While there are a number of studies demonstrating that infants possess perceptual color categories and a number demonstrating the effects of color language on categorical tasks in adults, there is disagreement about how language influences color categorization and how early in color word acquisition these effects emerge. Under one hypothesis, effects of language may occur online. In other words, if participants verbalize to themselves the names of colors during these tasks, language effects may emerge as soon as color terms are learned. Conversely, they may emerge gradually as children acquire extensive experience with color words. On one hand, Franklin, Clifford, Williamson, and Davies (2005) found that the categorical boundaries of young children were not influenced by their color word knowledge, suggesting that effects of language do not occur online and may emerge later after greater experience with color language. English-learning children who assigned the same label to two shade of blue (B1 and B2) and a different label to a shade of purple (P1) had the same categorical responses across the blue/green color boundary as children who had a mismatched color word boundary, assigning the same label to P1 and B2 and a different label to B1. In other words, children's incorrect color word categories were not predictive of their categorical responses on perceptual tasks. Instead, the standard category boundaries of adult speakers predicted their behaviors. Furthermore, among 
young children, Franklin et al. found no cross-linguistic differences on tests of categorical color perception. On the other hand, Goldstein, Davidoff and Roberson (2009) and Roberson, Davidoff, Davies and Shapiro (2004) tested a different color boundary and used a different metric for defining color word knowledge and consequently found evidence that color word knowledge does influence categorical color perception soon after color words are acquired. (See Franklin, Wright, \& Davies, 2009 for further discussion on this debate).

\section{Reconciling universalist and relativist accounts}

Moderate versions of the universalist and relativist accounts are not necessarily mutually exclusive. How can evidence for universalism in the form of pre-linguistic infant color categories be squared with studies which show effects of linguistic variation on color categorization and memory in adults? One possibility consistent with the presented data is that humans enter the world with universal perceptual constraints on color categories, which inform the boundaries and focal regions of linguistic color categories, without fully determining them. Where linguistic variability occurs, labels influence memory and processing time of colors. For further discussion see Kay and Regier (2007) and Regier, Kay, Gilbert and Ivry (2007).

On this view, once color labels are acquired, linguistic and perceptual color categorization systems may exist alongside each other. Alternatively, distinctions imposed by language could come to dominate color categorization. Consistent with the second account, Gilbert, Regier, Kay, and Ivry (2006) presented evidence of categorical perception of color only in the right visual field, which corresponds to the language dominant left hemisphere. Franklin et al., (2008) presented further evidence that left hemisphere dominance on categorical color tasks arises after children learn their color words. In fact, they found that prior to learning color words, categorical responding was stronger in the left visual field/right hemisphere. On the basis of this 
data, Franklin et al. (2008) argued that linguistically defined category boundaries may replace pre-linguistic categories.

\section{Color Word Acquisition}

As noted in the Introduction, early reports in the $20^{\text {th }}$ century found that children learned color words as late as age 8. Perhaps due to earlier instruction, children in the US now learn adult-like meanings for basic color words by 2 to 4 years of age (Shatz, Behrend, Gelman, \& Ebeling, 1996). Although children now acquire them earlier, researchers still frequently cite color words as a particularly difficult challenge. The primary evidence for this claim is that, similar to other difficult conceptual domains, like time, number, and emotion, children appear to use color words in a "haphazard and inconsistent" (p. 70, Pitchford \& Mullen, 2003) manner for months, sometimes years, before they master adult-like meanings.

Although we know that frequent input facilitates learning, relatively little is known about the mechanisms by which children converge on adult-like color word meanings. Children who attend preschool learn their color words more quickly than children who do not attend preschool (Shatz et al., 1996). Similarly, color words that appear more frequently in child-directed speech like green and blue are learned before less frequent color words like orange and gray (Yurovsky, Wagner, Barner \& Frank, 2015; Pitchford \& Mullen, 2005). However, beyond this somewhat uncontroversial observation that input frequency speeds learning, researchers have often disagreed about how color word learning works, and why it might pose a problem to young children.

Learning the meaning of a color word requires identifying at least two things. First, children must identify that the class of color words label color properties (i.e., surface reflectance) rather than size, shape or texture. This requires attending to color, abstracting it as a 
property independent of the objects on which they appear, and identifying color properties relevant to word meanings. Second, children must also identify the category boundaries of particular color words. Where does red end and orange begin? Both of these steps (identifying color properties and identifying category boundaries) have been put forward as potentials sources of difficulty in the acquisition process.

\section{Evidence for difficulty identifying color properties.}

With increasing evidence that infants possess perceptual color categories long before they acquire color word meanings, many current researchers have argued that these perceptual color categories may solve the category formation problem for children by strongly constraining the possible categories that could define color words meanings. As support for this argument, researchers have shown that infants exhibit categorical responses to changes that cross the blue/green, blue/purple and red/pink linguistic color boundaries of English-speaking adults. With the category boundary problem potentially addressed by the presence of pre-linguistic perceptual color categories, many have argued that children may learn the meanings of these words simply by mapping words onto their innately specified perceptual categories. For example, according to Shatz et al. (1996), “on perceptual tasks, infants treat the continuous dimension of hue categorically much as adults do... Thus, the apparent difficulty children have with color term acquisition cannot be primarily because the perceptual domain is continuous whereas the lexical domain is discontinuous"' (p. 178). Similarly, according to Pitchford and Mullen (2003), “Developmental studies have shown young children's perceptual color space is organized in a similar manner to that of the adult... Thus, when children engage in the learning of color terms, they already possess color percepts on which color concepts can be mapped"'. (p. 53). 
Due to the belief that the problem of identifying category boundaries may be solved by pre-linguistic perceptual color categories, much of the research in recent years has focused instead on the first problem, property identification or abstraction, as the primary source of difficulty in color word acquisition. Lending further plausibility to this idea, there is a general consensus among developmentalists that both infants and older children more readily categorize objects by shape than by color. Infants respond more to an object that makes an impossible change in shape or size at around four months but do not respond to a similar change in color until around eleven months of age (Wilcox, 1999). Such findings are taken as evidence that infants preferentially attend to shape over surface properties. Similarly, there is evidence that during the second year of life children exhibit a whole-object bias, which helps them quickly map new words onto referents (Macnamara, 1972; Markman, 1989). According to this proposal, the whole-object bias may direct children's attention to objects like rabbits rather than to their parts (e.g., legs, teeth, etc.), or properties (e.g., brown, soft). Consequently, the same attentional mechanisms that facilitate the acquisition of object labels may hinder the acquisition of object property labels like color words.

Consistent with this hypothesis, some studies have found that children fail to match objects by color until after they have already learned the meanings of color words (Sandhofer \& Smith, 1999; Kowalski \& Zimiles, 2006; Smith, 1984; for discussion, see Pitchford, 2006; Franklin, 2006). For example, Sandhofer and Smith (1999) showed children two objects with different shapes and the same color and then asked them to choose additional matches from a variety of objects with different shapes and different colors. They also intentionally avoided using any color terms during the matching task to prevent children who had learned some color words from employing this cue. Using a longitudinal method, they found that children were 
unable to match objects of the same color until after they had learned adult-like labels for colors. Based on this finding, they argued that color labels actually facilitate the abstraction of color by highlighting the similarities of color properties across exemplars. For example, a child might learn the word red by first hearing red cup and red ball and then abstracting the property that is shared across the two objects - i.e., the color red.

Other researchers, however, have argued that children readily abstract color but do not identify color as relevant to word meanings because of attentional constraints (for discussion, see Pitchford, 2006; Franklin, 2006). For example, Soja (1994) employed a variety of matching tasks and found that children who could not yet comprehend colors words could (1) learn a label for an animal that that was only distinguishable by color, and (2) choose which of two differently shaped objects was a color match for a third differently shaped object. Employing a similar logic, Wilcox, Woods, and Chapa (2008) found a similar ability to categorize according to color in preverbal infants. They found that although infants generally categorize objects by shape more readily than by color, when infants receive training which indicates that color, but not shape, predicts the function of an object, they are able to categorize by color over shape at a younger age, around 7 months (Wilcox, Woods \& Chapa, 2008; Wilcox \& Chapa, 2004). Without training, they found this ability does not emerge until around 11 months of age (Wilcox, 1999). Based on such results, Soja (1994) and others (O'Hanlon \& Roberson, 2006; Sandhofer \& Smith, 2001; Pitchford \& Mullen, 2002) have argued that children abstract the dimension of color prior to understanding color words, and that linguistic constraints like the whole object constraint and mutual exclusivity make color word learning hard, rather than the problem of abstracting color, per se (though see Sandhofer \& Smith, 1999, for an argument that Soja's task did not require color abstraction). 
COLOR WORDS 21

Together, existing evidence suggests that although children have difficulty identifying color properties as likely candidates for word meanings, this is not because they can't abstract color prior to learning labels. Children readily abstract color when it is relevant to categorization and can use such properties to learn new labels.

\section{Evidence for difficulties with category formation}

The above explanations share an assumption that children have difficulty identifying color as relevant to word meaning, either because of an inability to abstract color or because of a bias to preferentially attend to other properties. However, in addition to identifying the relevant dimension of experience, children must also identify adult-like boundaries for each color word.

Although there is increasing evidence that infants possess pre-linguistic perceptual color categories that could constrain word learning, there are also good reasons to believe that the acquisition of color words cannot be reduced to a simple problem of mapping words onto these categories. Rather, it must also involve a learning process wherein children's initial mappings are refined based on language-specific experience. Perhaps most important is evidence that languages differ substantially in how they carve up perceptual color space. Although the World Color Survey revealed that there are strong cross-linguistic constraints on the locations of color word boundaries, languages still vary substantially both in the number of basic color words they have (from 2 to 12) and in how these words divide up color space.

According to the World Color Survey, languages that feature only two or three color words organize color space in a way unlike languages with more color words, frequently grouping warm colors (e.g., white, red, yellow) under one label and cool colors (e.g., black, green, blue) under another. For example, two of the five linguistic color categories used in the OtjiHerero language, a language spoken by the Himba, an ethnic group in rural Namibia, are 
burou (green, blue and purple) and dumbu (beige, yellow and light green). Thus, OtjiHerero marks at least one color boundary that is absent in English, while failing to mark others. Differences like these are not explained by differences in the number of color words that a language provides. Data from the World Color Survey (Kay et al., 2009) show that some languages that have four basic color terms mark a category boundary between red and yellow (e.g., Culina, spoken in Peru; Waorini, spoken in Ecuador) whereas others do not (e.g., Chácobo, spoken in Bolivia; Múra-Pirahã, spoken in Brazil). In sum, even if infants perceive color like adults and use pre-linguistic categories as inputs to learning, the categories encoded by language cannot be fully determined by perception. This gap between perception and language suggests that inductive learning - i.e., a process of constructing categories from experience with a subset of possible exemplars - must play a significant role.

Recently, Wagner, Dobkins and Barner (2013) examined this idea by testing children who have begun using color words, but do not yet have adult-like meanings for them. According to past accounts, such children struggle to learn color word meanings because of abstraction - or perhaps because they preferentially attend to other dimensions of meaning like shape. According to this hypothesis, when children initially begin using color words, they should apply them randomly to objects. However, if children's main problem is category induction, rather than abstraction, then they may exhibit preliminary, non-adult, meanings for color words early in acquisition, and therefore respond non-randomly when labeling objects.

Wagner et al. (2013) found that from the time they begin saying color words, perceptually adjacent color labeling errors are more common than perceptually distant ones (for similar evidence see Pitchford \& Mullen, 2003; Carey \& Bartlett, 1978; Bartlett, 1978; Nagel, 1906). They also found that children were likely to repeat the same errors across different trials - 
e.g., that if they used a color word like red to label an orange or yellow stimulus, they were very likely ( $76 \%$ of the time) to also use red to label red stimuli. In general, if children used color words, they did so systematically, rather than randomly. Similar systematic errors were also observed in an easier test of color word comprehension that did not require children to generate labels.

Based on these data, Wagner, Dobkins, and Barner (2013) argued that even if children have a difficult time identifying color as relevant to word meaning, they appear to solve this piece of the puzzle very early in development, and at least by the time they begin producing color words in speech. Thus, the long delay between the onset of color word use and the formation of adult-like meanings may result not from difficulties identifying color as relevant to word meaning but instead from a gradual inductive process of acquiring language-specific color word boundaries. During this period, as children acquire new color words, the newly acquired color words constrain the meanings of previously acquired color words. For example, a child who initially treats blue as a label for both blue and purple may restrict its use to only blue upon learning the word purple. Consistent with this, O'Hanlon \& Roberson (2006) found that children are faster to learn a new color word for a particular color stimulus if the new term is contrasted with the child's previously used, preliminary label for that stimulus, e.g., this is NOT blue, it's purple. Learning environments that contain multiple objects which contrast in color have also been shown to accelerate learning (e.g., Waxman \& Klibanoff, 2000; Sandhofer \& Smith, 2001), providing further support that learning how color words contrast with each other is a critical part of the color word acquisition process.

\section{Color words meanings continue to be refined for several years}

The studies presented thus far are consistent with the idea that children abstract color as 
relevant to the meanings of color words early in acquisition, and struggle to identify the precise boundaries of these words despite the existence of prelinguistic perceptual color categories. Initially, children appear to overextend a small number of highly frequent words like red, green, and blue to label adjacent colors like orange, purple, pink, etc. Consistent with this hypothesis, additional studies suggest that children take many months or years to learn the precise boundaries of color words even after they have identified the focal color that they label. As evidence for this, studies by Raskin, Maital and Bornstein (1983) and Saji, Asano, Oishi and Imai (2015) compared the boundaries of color word meanings in children to adults and found that children's boundaries are wider and overlap more than adults. For example, Saji et al. (2015) asked children to label an array of color chips and found that their color word boundaries overlapped more than those of adults until age five, despite the fact that the children correctly labeled the focal regions of each category by around three years of age.

Similarly, Mervis, Catlin and Rosch (1975) found that the color word boundaries of children differ from those of adults up to age eight. Specifically, they found that, along the dimension of hue (what distinguishes purple from blue from green), adults possessed more narrow categories than eight-year-olds, a finding consistent with the idea that very young children's categories are very broad and overlapping. However, interestingly, Mervis et al. also found that adults actually have broader categories along the dimensions of brightness and saturation (which respectively distinguish a light red from a dark red and a vibrant red from a dull red). Unlike adults, eight-year-olds and five-year-olds excluded colors low in saturation like grayish blue or brownish red from their chromatic categories like blue or red.

These are particularly interesting in light of the trajectory of color language evolution described by the World Color Survey. Specifically, Kay et al. (2009) suggested that languages 
which feature only a few color terms typically prefer to encode a light vs. dark distinction first, and second prefer chromatic terms that span multiple English color categories, oftentimes excluding the lightest and darkest shades of those hues. In this way, the patterns observed in languages with only a few color terms are similar to those observed in children by Mervis, Catlin and Rosch (1975) who excluded light and dark shades from their meanings for chromatic English color words like blue or red.

\section{Conclusion}

As in many other domains of word learning such as time, number, and space (e.g., Widen \& Russell, 2003; Tillman \& Barner, 2015; Shatz, Tare, Nguyen \& Young, 2010; Ameel, Malt \& Storms, 2008; for review see Wagner, Tillman \& Barner, 2017), children appear to produce color words for many months before converging on adult-like meanings. Recent research indicates that during this period, children rely on their linguistic input to solve a complex problem: Where does their language draw the boundaries between color words? Like in many domains, this is a slow process that can take years and likely requires learning how words within a class contrast with one another (for review, see Wagner, Tillman, \& Barner, 2017).

There are still many open questions in the field of color word acquisition. First, if children can abstract color concepts early in development, how do they accomplish this task? Second, how do children converge on language-specific boundaries and what role do prelinguistic perceptual categories and linguistic input play in this process? Cross-linguistic variability in color word boundaries eliminates the possibility that color word meanings are directly mapped onto perceptual categories. However, these categories may nevertheless provide children with strong constraints on color word meanings. Similarly, while current research increasingly supports that notion that color categories receive input from both the perceptual and 
COLOR WORDS 26

linguistic systems, further research is required to clarify how these systems interact. Do linguistic color categories replace perceptual ones after color words are acquired (Franklin et al, 2008), do they alter perceptual categories (e.g., Goldstein, Davidoff \& Roberson, 2009), or do they simply exist in parallel alongside perceptual categories (e.g., Franklin et al., 2005). Future work should explore these questions, to reveal how language and perception conspire to represent color. 
COLOR WORDS 27

\section{References}

Ameel, E., Malt, B, \& Storms, G. (2008). Object naming and later lexical development: From baby bottle to beer bottle. Journal of Memory and Language, 58, 262-285.

Barner, D., Li, P., \& Snedeker, J. (2010). Words as windows to thought: The case of object representation. Current Directions in Psychological Science, 19(3), 195-200.

Bartlett, E. J. (1978). The acquisition of the meaning of color terms: A study of lexical development. In R. N. Campbell \& P. T. Smith (Eds.), Recent Advances in the Psychology of Language (pp. 89-108). New York: Plenum.

Bateman, W. (1915). The naming of colors by Children the Binet Test. The Pedagogical Seminary, 22, 469-486.

Berlin, B. \& Kay, P. (1969). Basic Color Terms: Their Universality and Evolution. Berkeley, CA: University of California Press.

Boothe, R., Dobson, V. \& Teller, D. (1985). Postnatal development of vision in human and nonhuman primates. Ann. Rev. Neurosci., 8, 495-545.

Bornstein, M., Kessen, W., Weiskopf, S. (1976). Color vision and hue categorization in young human infants. Journal of Experimental Psychology: Human Perception and Performance, 2, 115-129.

Briscoe, A., \& Chittka, L. (2001). The evolution of color vision in insects. Annual Review of Entomology, 46, 471-510.

Carey, S., \& Bartlett, E. (1978). Acquiring a single new word. Proceedings of the Stanford Child Language Conference, 15, 17-29.

Cronin, T. \& Marshall, J. (1989). A retina with at least ten spectral types of photoreceptors in a mantis shrimp. Nature, 339, 137-140 
Cronin, T. W., Bok, M. J., Marshall, N. J., \& Caldwell, R. L. (2014). Filtering and polychromatic vision in mantis shrimps: themes in visible and ultraviolet vision. Philosophical Transactions of the Royal Society B: Biological Sciences, 369(1636), 20130032.

Curcio, C. A., Allen, K. A., Sloan, K. R., Lerea, C. L., Hurley, J. B., Klock, I. B. \& Milam, A. H. (1991) Distribution and morphology of human cone photoreceptors stained with anti-blue opsin. The Journal of Comparitive Neurology, 312, 610-624.

Dannemiller, J. L. (1989). A test of color constancy in 9- and 20-week-old human infants following simulated illuminant changes. Developmental Psychology, 25, 171-184.

Dobson \& Teller (1978). Visual acuity in human infants: A review and comparison of behavioral and electrophysiological studies. Vision Research, 18, 1469-1483.

Eimas, P., Siqueland, E., Jusczyk, P., \& Vigorito, J. (1971). Speech perception in infants. Science, 171 (968), 303-6.

Franklin, A. (2006). Constraints on children's color term acquisition. Journal of Experimental Child Psychology, 94, 322-327.

Franklin, A. \& Davies, I. RL. (2004). New evidence for infant colour categories. British Journal of Developmental Psychology, 22(3). 349-277.

Franklin, Wright, and Davies (2009) What can we learn from toddlers about categorical perception of color? Comments on Goldstein, Davidoff, and Roberson. Journal of Experimental Child Psychology, 102 (2). pp. 239-245.

Franklin, A., Clifford, A., Williamson, E. \& Davies, I. (2005). Colour term knowledge does not affect categorical perception of colour in toddlers. Journal of Experimental Child Psychology, 90, 114-141. 
Franklin, A., Drivonikou, G., Clifford, A, Kay, P., Regier, T. \& Davies, I. (2008).

Lateralization of categorical perception of color changes with color term acquisition. Proceedings of the National Academy of Science, 105, 18222-18225.

Franklin, A., Pilling, M. \& Davies, I. (2005). The nature of infant color categorization: Evidence from eye movements on a target detection task. Journal of Experimental Child Psychology, 91, 227-248.

Gilbert, A. L., Regier, T., Kay, P., \& Ivry, R. B. (2006). Whorf hypothesis is supported in the right visual field but not the left. Proceedings of the National Academy of Sciences of the United States of America, 103(2), 489-494.

Goldstein, J., Davidoff, J., \& Roberson, D. (2009) Knowing color terms enhances recognition: Further evidence from English \& Himba. Journal of Experimental Child Psychology, $102,219-238$.

Goris, R. (2011). Infrared organs of snakes: An integral part of vision. Journal of Herpetology, $45,2-14$.

Gussoni E., Pavlath G.K., Lanctot A.M., et al. (1992). Normal dystrophin transcripts detected in Duchenne muscular dystrophy patients after myoblast transplantation. Nature 356, $435-438$

Heider, E. R. \& Olivier, D. C. (1972). The structure of the color space in naming and memory for two languages. Cognitive Psychology, 3, 337-354.

Heider, E. R. (1972). Universals in color naming and memory. Journal of Experimental Psychology, 93(1), 10-20

Hofer, H., Carroll, J., Neitz, J., Neitz, M., \& Wiliams, D. R. (2005). Organization of the human trichomatic cone mosaic. The Journal of Neuroscience, 25(42), 9669-9679 
Jacobs, G. (2009). Evolution of colour vision in mammals. Philosophical Transactions of the Royal Society B, 364, 2957-2967.

Kay \& Regier (2007). Color naming universals: The case of Berinmo. Cognition, 102, 289298.

Kay, P., Berlin, B., Maffi, L., Merrfield, W. R. \& Cook, R (2009). The World Color Survey. Palo Alto, CA: CSLI Press.

Knoblauch, K., Vital-Durand, F., \& Barbur, J. (2001). Variation of chromatic sensitivity across the life span. Vision Research, 41, 23-36.

Kowalski \& Zimiles (2006). The relation between children's conceptual functioning with color and color term acquisition. Journal of Experimental Child Psychology, 94, 301-321.

MacNamara, J. (1972). Cognitive basis of language learning in infants. Psychological Review, $79(1), 1-13$.

Markman, E. M. (1989). Categorization and Naming in Children: Problems of Induction. Cambridge, MA: MIT Press.

Marshall, J. \& Oberwinkler, J. (1999). The colourful world of the mantis shrimp. Nature, 401, 873-874.

Mervis, C. B., Catlin, J \& Rosch, E. (1975). Development of the structure of color categories. Developmental Psychology, 11(1), 54-60.

Nagel, W. A. (1906). Observations on the color-sense of a child. Journal of Comparative Neurology and Psychology, 16, 217-230.

O’Hanlon, C., \& Roberson, D. (2006). Learning in context: Linguistic and attentional constraints on children's color term learning. Journal of Experimental Child Psychology, 94, 275-300. 
COLOR WORDS 31

Pilling, M., Wiggett, A., Ozgen, E., \& Davies, I.R. (2003). Is color "categorical perception" really perceptual? Memory and Cognition, 31(4), 538-551

Pitchford, N. \& Mullen, K. (2002). Is the acquisition of basic-colour terms in young children constrained? Perception, 31, 1349-1370.

Pitchford, N., \& Mullen, K. (2003). The development of conceptual colour categories in preschool children: Influence of perception on categorization. Visual Cognition, 10, 51-77

Pitchford, N., \& Mullen, K. (2005). The role of perception, language, and preference in the development of basic color terms. Journal of Experimental Child Psychology, 90, 275302.

Pitchford, N. (2006). Reflections on how color term acquisition is constrained. Journal of Experimental Child Psychology, 94, 328-333.

Raskin, L., Maital, S., \& Bornstein, M. (1983). Perceptual categorization of color: A life-span study. Psychological Research, 45, 135-145.

Regier, T., Kay, P., Gilbert, A. L., \& Ivry, R. B. (2007). Language and thought: Which side are you on, anyway? In B. Malt and P. Wolff (Eds.), Words and the Mind: How Words Capture Human Experience (pp. 165-182). New York: Oxford University Press.

Roberson, D. \& Davidoff, J. (2000). The categorical perception of colors and facial expressions: The effect of verbal interference. Memory and Cognition, 28(2000), 977986.

Roberson, D., Davidoff, J., Davies, I., \& Shapiro, L. (2005). Color categories: Evidence for the cultural relativity hypothesis. Cognitive Psychology, 50, 378-411.

Roberson, D., Davies, I.R.L., \& Davidoff, J. (2000). Color categories are not universal: Replications and new evidence from a Stone Age culture. Journal of Experimental 
Psychology: General, 129(2000), 369-398.

Roberson, D., Hanley, J.R. \& Pak, H. (2009). Thresholds for color discrimination in English and Korean speakers. Cognition, 112, 482-487.

Roorda, A. \& Williamsn, D. R. (1999). The arrangement of the three cone classes in the living human eye. Nature, 379, 520-522.

Saji, N., Asano, M., Oishi, M., \& Imai, M. (2015). How do children construct the color lexicon? : Restructuring the domain as a connected system. Proceedings of the 37th Annual Meeting of the Cognitive Science Society.

Sandhofer, C. \& Smith, L. (1999). Learning color words involves learning a system of mappings. Developmental Psychology, 35, 668-679.

Sandhofer, C. \& Smith, L. (2001). Why children learn color and size words so differently: Evidence from Adults' learning of artificial terms. Journal of Experimental Psychology: General, 130 (4), 600-620.

Shatz, M., Behrend, D., Gelman, S., \& Ebeling, K. (1996). Colour term knowledge in twoyear-olds: Evidence for early competence. Journal of Child Language, 23, 177-199.

Shatz, M., Tare, M., Nguyen, S. P., \& Young, T. (2010). Acquiring non-object terms: The case for time words. Journal of Cognition and Development, 11, 16-36.

Simunovic, M. (2010). Color vision deficiency. Eye, 24, 747-755.

Smith, L. B. (1984). Young children's understanding of attributes and dimensions: A comparison of conceptual and linguistic measures. Child Development, 55, 363-380

Soja, N. (1994). Young children's concept of color and its relation to the acquisition of color words. Child Development, 65, 918-937.

Teller, D. \& Bornstein, M. (1985) Infant color vision. In ed. P. Salapatek \& L. Cohen 
Handbook of Infant Perception. (pp. 185-236). Orlando, FL: Academic Press.

Tillman, K.; and Barner, D. (2015). Learning the language of time: Children's acquisition of duration words. Cognitive Psychology, 78: 57-77.

Wagner, K., Dobkins, K. \& Barner, D. (2013). Slow mapping: Color word learning as a gradual inductive process. Cognition (127), 307-317.

Wagner, K.; Tillman, K.; and Barner, D. (To Appear) Inferring number, time, and color concepts from core knowledge and linguistic structure. In Barner, D.; and Baron, A. S., editor(s), Core Knowledge \& Conceptual Change. Oxford University Press, 2017.

Wässle, H. (2004). Parallel processing in the mammalian retina. Nature Reviews Neuroscience, 5, 1-11.

Waxman, S. \& Klibanoff, R. (2000). The role of comparison in the extension of novel adjectives. Developmental Psychology, 36, 571-581.

Werker, J., Gilbert, J., Humphrey, K., \& Tees, R. (1981). Developmental aspects of crosslanguage speech perception. Child Development, 52 (1), 349-55.

Widen, S., \& Russell, J. (2003). A closer look at preschoolers' freely produced labels for facial expressions. Developmental Psychology, 39, 114-128.

Wilcox, T. (1999). Object individuation: Infants' use of shape, size, pattern, and color. Cognition, 72, 125-166.

Wilcox, T., \& Chapa, C. (2004). Priming infants to attend to color and pattern information in an individuation task. Cognition, 90, 265-302.

Wilcox, T., Woods, R., \& Chapa, C. (2008). Color-functiono categories that prime infants to use color information in an object individuation task. Cognitive Psychology, 57, 220261 
Winawer, J., Witthoft, N., Frank, M., Wu, L., \& Boroditsky, L. (2007). Russian blues reveal effects of language on color discrimination. Proceedings of the National Academy of Sciences, 104, 7780-5.

Yang, J., Kanazawa, S., Yamaguchi, M., \& Ichiro, K. (2013). Journal of Experimental Child Psychology, 115, 126-136.

Yurovsky, D., Wagner, K., Barner, D., \& Frank, M. (2015). Signatures of domain-general categorization mechanisms in color word learning. Proceedings of the 37th Annual Conference of the Cognitive Science Society, Pasadena, CA

\section{Further Reading}

Carey, S., \& Bartlett, E. (1978). Acquiring a single new word. Proceedings of the Stanford Child Language Conference, 15, 17-29.

Franklin, A. (2006). Constraints on children's color term acquisition. Journal of Experimental Child Psychology, 94, 322-327.

Franklin, A. \& Davies, I. RL. (2004). New evidence for infant colour categories. British Journal of Developmental Psychology, 22(3). 349-277.

Heider, E. R. (1972). Universals in color naming and memory. Journal of Experimental Psychology, 93(1), 10-20

Kay, P., Berlin, B., Maffi, L., Merrfield, W. R. \& Cook, R (2009). The World Color Survey. Palo Alto, CA: CSLI Press.

Kowalski \& Zimiles (2006). The relation between children's conceptual functioning with color and color term acquisition. Journal of Experimental Child Psychology, 94, 301-321.

O’Hanlon, C., \& Roberson, D. (2006). Learning in context: Linguistic and attentional 
COLOR WORDS 35

constraints on children's color term learning. Journal of Experimental Child Psychology, 94, 275-300.

Regier, T., Kay, P., Gilbert, A. L., \& Ivry, R. B. (2007). Language and thought: Which side are you on, anyway? In B. Malt and P. Wolff(Eds.), Words and the Mind: How Words Capture Human Experience (pp. 165-182). New York: Oxford University Press.

Roberson, D., Hanley, J.R. \& Pak, H. (2009). Thresholds for color discrimination in English and Korean speakers. Cognition, 112, 482-487.

Sandhofer, C. \& Smith, L. (2001). Why children learn color and size words so differently: Evidence from Adults' learning of artificial terms. Journal of Experimental Psychology: General, 130 (4), 600-620.

Teller, D. \& Bornstein, M. (1985) Infant color vision. In ed. P. Salapatek \& L. Cohen Handbook of Infant Perception. (pp. 185-236). Orlando, FL: Academic Press.

Pitchford, N. (2006). Reflections on how color term acquisition is constrained. Journal of Experimental Child Psychology, 94, 328-333.

Pitchford, N., \& Mullen, K. (2003). The development of conceptual colour categories in preschool children: Influence of perception on categorization. Visual Cognition, 10, 51-77

Roberson, D., Davidoff, J., Davies, I., \& Shapiro, L. (2005). Color categories: Evidence for the cultural relativity hypothesis. Cognitive Psychology, 50, 378-411.

Wagner, K., Dobkins, K. \& Barner, D. (2013). Slow mapping: Color word learning as a gradual inductive process. Cognition (127), 307-317.

Winawer, J., Witthoft, N., Frank, M., Wu, L., \& Boroditsky, L. (2007). Russian blues reveal effects of language on color discrimination. Proceedings of the National Academy of Sciences, 104, 7780-5. 
COLOR WORDS 36 